\title{
Overexpression of Strigolactone-Associated Genes Exerts Fine-Tuning Selection on Soybean Rhizosphere Bacterial and Fungal Microbiome
}

\author{
Fang Liu, ${ }^{1}$ John Hollis Rice, ${ }^{2}$ Valeria Lopes, ${ }^{2}$ Parwinder Grewal, ${ }^{3}$ Sarah L. Lebeis, ${ }^{4}$ Tarek Hewezi, ${ }^{2}$ and Margaret E. Staton ${ }^{1, \dagger}$ \\ ${ }^{1}$ Department of Entomology and Plant Pathology, University of Tennessee, Knoxville, TN 37996 \\ ${ }^{2}$ Department of Plant Science, University of Tennessee, Knoxville, TN 37996 \\ ${ }^{3}$ College of Science, University of Texas Rio Grande Valley, Edinburg, TX 78539 \\ ${ }^{4}$ Department of Microbiology, University of Tennessee, Knoxville, TN 37996
}

Accepted for publication 15 April 2020.

\section{ABSTRACT}

Strigolactones are a recently discovered class of carotenoid-derived plant hormones with a wide variety of functions, including acting as signaling molecules in the rhizosphere to promote arbuscular mycorrhizal fungi colonization and parasitic seed germination. To determine whether strigolactones influence the recruitment of microbes to the rhizosphere, we characterized both bacterial and fungal communities in response to the overexpression of genes involved in strigolactone biosynthesis (MAX1d) and signaling perception (D14 and MAX2a) in soybean (Glycine max). The amplicon sequencing-based results suggest that strigolactone overexpression lines had altered soybean rhizosphere bacteria composition at both the community level and individual taxa level with genera including Shinella and Bdellovibrio consistently more abundant across all three overexpression constructs. In addition, the responses of the soybean rhizosphere bacteria community differed significantly across the genes, with lines overexpressing genes involved in strigolactone biosynthesis (MAX1d) yielding a divergent bacterial community in comparison with those with altered expression of strigolactone perception genes (D14 and MAX2a). The overexpressed genes did not significantly impact the overall fungal community distribution; however, some individual taxa were altered in abundance. MAX1d and D14 overexpression lines had significantly enriched abundance of Fusarium solani. The mediating role of strigolactone biosynthesis and signaling genes on soybean rhizosphere bacterial and fungal communities confirmed strigolactone's importance in the rhizosphere host-microbe communication and microbial community structure.

Keywords: bacteria, crop, fungi, metagenomics, microbiome, rhizosphere and phyllosphere, soybean, strigolactone, transformation
${ }^{\dagger}$ Corresponding author: M. Staton; mstaton1@utk.edu

Author contributions: F.L. led the experimental and genomic investigations, including methodology, validation, formal analysis, and writing of the original draft. J.H.R. and F.L. helped to perform some experiments and collect data. T.H. and S.L. contributed to conceptualization and methodology of the experiments and also contributed resources and supervision. P.G. contributed to conceptualization of the research and initial funding acquisition. M.S. contributed to conceptualization and methodology of the experiments and also provided supervision and led funding acquisition. All authors reviewed and edited the final manuscript.

Funding: This work was funded by the Tennessee Soybean Promotion Board (\#16120-P, \#17-120-R, and \#18-120-R) and also by the University of Tennessee Institute of Agriculture. The funding bodies played no role in the design of the study or collection, analysis, and interpretation of data or in writing the manuscript. Fang Liu's stipend while studying at the Department of Entomology and Plant Pathology, University of Tennessee, Knoxville was sponsored by the China Scholarship Council.

*The $e$-Xtra logo stands for "electronic extra" and indicates that one supplementary file, two supplementary figures, and three supplementary tables are published online.

The author(s) declare no conflict of interest.
Plants have evolved intimate interactions with their associated microbiomes for various functions, including nutrient uptake, stress tolerance, pathogen defense, and phenotypic plasticity, all facilitated by diverse plant-growth-promoting microbes (Bakker et al. 2018; Goh et al. 2013). Comprehensive and mechanistic understanding of plant microbiome assembly is crucial for optimizing beneficial plant-microbe interactions and maximizing the benefits offered by the plant microbiome for agricultural applications (Pieterse et al. 2016). The rhizosphere (the narrow region between the plant root and attached soil) is a dynamic and active interface characterized by intensive communication between the plant and microbes (Philippot et al. 2013). In this zone, plants actively and dynamically modulate the composition and function of microbes via root exudates, secondary metabolites, or specific signaling molecules in response to changing soil biotic or abiotic stimuli (Berendsen et al. 2018; Castrillo et al. 2017; Lebeis et al. 2015; Stringlis et al. 2018; Xu et al. 2018; Zhalnina et al. 2018).

Strigolactones are a group of carotenoid-derived plant hormones and signaling molecules involved in plant-microbe interactions (Al-Babili and Bouwmeester 2015; Cook et al. 1966). The 
biosynthesis of strigolactones starts with the activity of a $\beta$-carotene isomerase DWARF27 (D27) and two carotenoid cleavage dioxygenases (CCD7 and CCD8) to produce carlactone (Waters et al. 2017). Carlactones are then catalyzed by a cytochrome P450 enzyme called more axillary growth 1 (MAX1) and subsequent uncharacterized enzymes to produce either canonical or noncanonical strigolactones (López-Ráez et al. 2017; Waters et al. 2017). Expression of genes involved in strigolactone biosynthesis is highest in plant roots (e.g., rice, Arabidopsis, sorghum, and pea) and low or undetectable in other tissues (Al-Babili and Bouwmeester 2015; Ruyter-Spira et al. 2013; Yoneyama et al. 2007). The synthesized strigolactones function as plant hormones via downstream

TABLE 1

Genes participating in strigolactone biosynthesis and perception in Arabidopsis and Williams 82

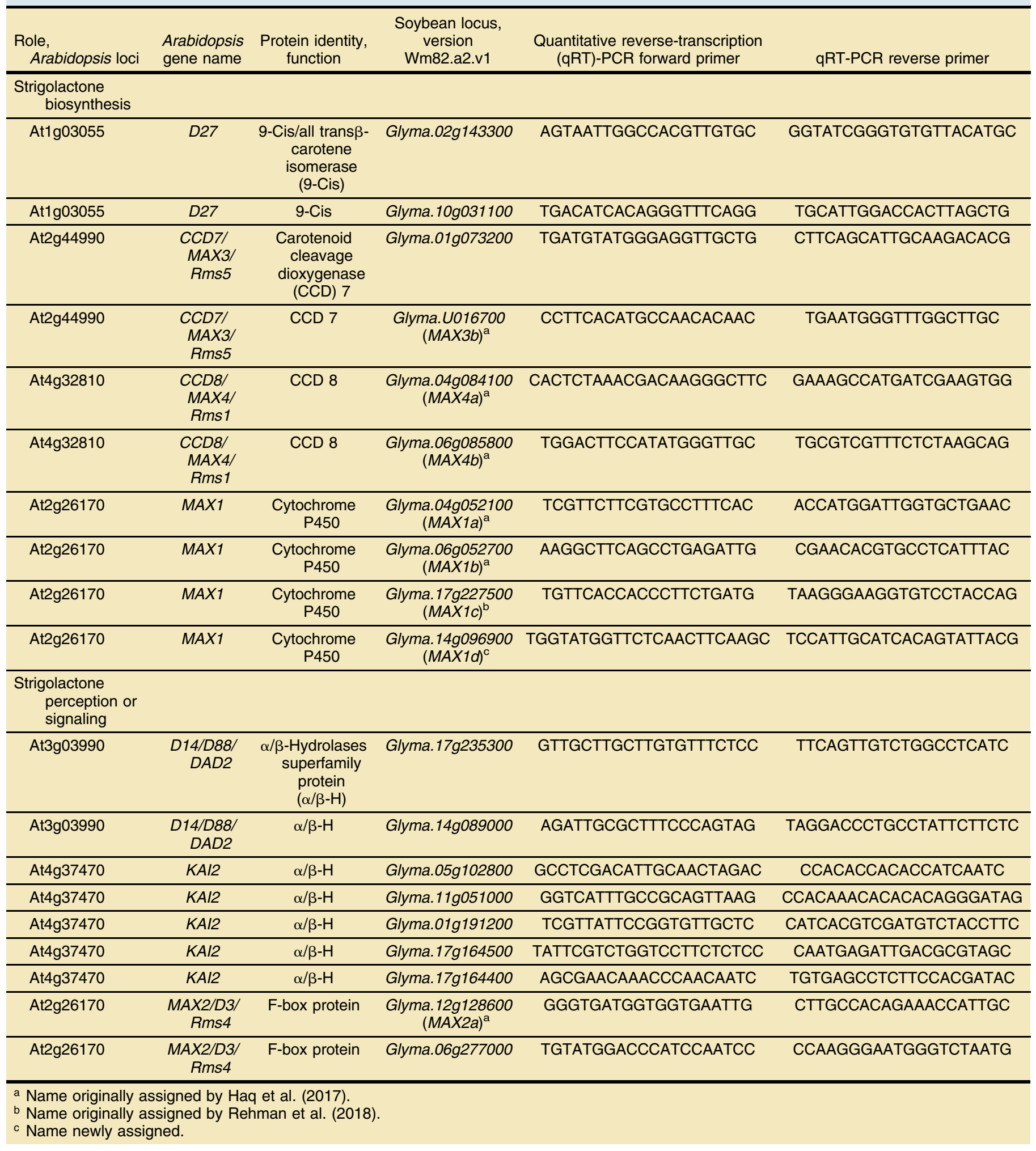


signaling transduction networks enabled by a/ $\beta$-fold hydrolase $D 14$ and F-box protein Max2 (Arite et al. 2009; Beveridge and Kyozuka 2010; Nelson et al. 2011). Simultaneously, strigolactones are exuded into the rhizosphere; however, transport mechanisms are not yet well understood, with the only characterized transport gene, the ABC transporter PDR1, first identified in Petunia hybrida (Kretzschmar et al. 2012).

In the rhizosphere, strigolactones promote root colonization by symbiotic arbuscular mycorrhizal fungi (AMF) in many species, including Lotus japonicus, petunia, sorghum, tomato, and Fabaceae plants (Akiyama et al. 2005; Besserer et al. 2006; Kretzschmar et al. 2012; Yoneyama et al. 2008). Their presence is also perceived as germination stimulants for root-parasitic plants of the family Orobanchaceae (Yoneyama et al. 2008). Strigolactones have recently been suggested to function as important modulators for legume nodulation. In soybean, mutations of strigolactone biosynthesis genes have yielded nodulation phenotypes. Overexpression of $M A X 3 b(\mathrm{CCD} 7)$ increased nodule number per gram of healthy root while knockdown of the expression did the opposite (Haq et al. 2017). Knockdowns of MAX1a (MAX1) and MAX4a (CCD8) also exhibited decreased nodulation (Rehman et al. 2018). Both studies found nodule-related gene expression differences and altered hormone levels in addition to the phenotypic effects (Haq et al. 2017; Rehman et al. 2018). In both pea and alfalfa, in vitro application of the synthetic strigolactone analog GR2 $4^{\mathrm{rac}}$ increased the number of nodules per plant (Foo and Davies 2011; Soto et al. 2010). Furthermore, reduced nodule numbers were detected in the strigolactone synthesis mutants $c c d 7$ and $c c d 8$ in comparison with wild-type pea, and this reduction of nodule formation could be rescued by GR24 ${ }^{\text {rac }}$ to a similar level as the wild type (Foo and Davies 2011; Foo et al. 2013). However, promotion of nodulation by strigolactones does not appear to be due to their stimulatory effects on rhizobia growth or increased expression of nod genes (Foo and Davies 2011; Moscatiello et al. 2010; Soto et al. 2010). One study found that strigolactone application enhanced surface motility of Sinorhizobium meliloti, a rhizobia of alfalfa (PeláezVico et al. 2016), while another indicated that it promoted infection thread formation (McAdam et al. 2017), providing a number of possible mechanisms for strigolactones to help establish rhizobia in nodules through direct influence of the microbial partner.

The role that strigolactones play as signaling molecules for both AMF and nodule-associated microbes suggests that they may also have a broader role in mediating the overall rhizosphere microbiome. In Arabidopsis, lack of strigolactone production, induced through MAX4-deficient mutants, altered the fungal community composition in the rhizosphere but did not influence the bacterial community (Carvalhais et al. 2019). In contrast, rice mutants in strigolactone production (d17) and signaling (d14) demonstrated strong differences in the rhizosphere bacterial community composition but less noticeable impacts on fungi (Nasir et al. 2019). To date, the role of strigolactones in shaping the rhizosphere microbiome has yet to be examined in a legume. We hypothesize that strigolactone production and signaling in soybean roots directly or indirectly mediate microbial recruitment to the rhizosphere. To explore this question, we generated transgenic hairy root plants overexpressing MAX1d, MAX2a, and D14 genes and used amplicon sequencing to characterize the resulting differences of both bacterial and fungal communities in the rhizosphere.

\section{MATERIALS AND METHODS}

Candidate gene selection. Genes involved in strigolactone biosynthesis and perception in Arabidopsis thaliana were obtained from the TAIR database (Berardini et al. 2015). Corresponding homologs in Glycine max were retrieved from Phytozome (Goodstein et al. 2012) (Table 1; Fig. 1) and previous publications (Haq et al. 2017; Rehman et al. 2018). In total, 19 G. max genes were identified. The name MAXld was assigned to Glyma.14g096900 based on a recent phylogenetic study (Rehman et al. 2018), in which four MAX1 homologs were identified in G. max, with three of them explicitly named MAXIa, MAX1b, and MAX1c. The mRNA sequence of each homolog was downloaded from SoyBase (Grant et al. 2010) and multiple sequence alignments of gene family members were created with Clustal Omega (Sievers and Higgins 2018). Genespecific primers were designed from sequence regions that are unique to each gene using the Primer3 (Untergasser et al. 2012) online tool. To quantify the relative expression level for each gene, total RNA was extracted from root tissues of Williams 82 plants with Trizol reagent and subsequently purified using chloroform extraction. After DNase treatment, the DNA-free RNA was used for gene quantification assays using one-step quantitative reverse transcription-polymerase chain reaction (qRT-PCR) (Power SYBR Green RNA-to-CT 1-Step Kit). The qPCR assays were conducted using three biological samples, each with three technical replicates on an ABI 7900HT Fast Real-Time PCR System (Applied Biosystems). The soybean ubiquitin gene (Glyma.20G141600) was used as a constitutively expressed reference gene. Quantification of gene expression levels was performed as previously described (Rambani et al. 2015).

Construction of overexpression binary vectors and generation of transgenic hairy roots. The coding sequences of MAXId (Glyma.14g096900), D14 (Glyma.17g235300), and MAX2a (Glyma.12g128600) genes were amplified from Williams 82 root cDNA using gene-specific primers containing AscI and BamHI restriction sites (Supplementary Table S1). Specifically, $10.5 \mu \mathrm{l}$ of DNA-free RNA $(50 \mathrm{ng} / \mu \mathrm{l})$ and $0.5 \mu \mathrm{l}$ of poly-dT $(50 \mu \mathrm{M})$ were used to synthesize the first-strand cDNA. The RNA and poly-dT mixture was heated at $70^{\circ} \mathrm{C}$ for $5 \mathrm{~min}$, then transferred to ice for $4 \mathrm{~min}$, where $4 \mu \mathrm{l}$ of first-strand buffer $(5 \times), 2 \mu \mathrm{l}$ of dNTP mix $(10 \mathrm{mM})$, $2 \mu \mathrm{l}$ of dithiothreitol $(100 \mathrm{mM})$, and $1 \mu \mathrm{l}$ of smart Moloney murine leukemia virus reverse transcription (Clontech) were added to the

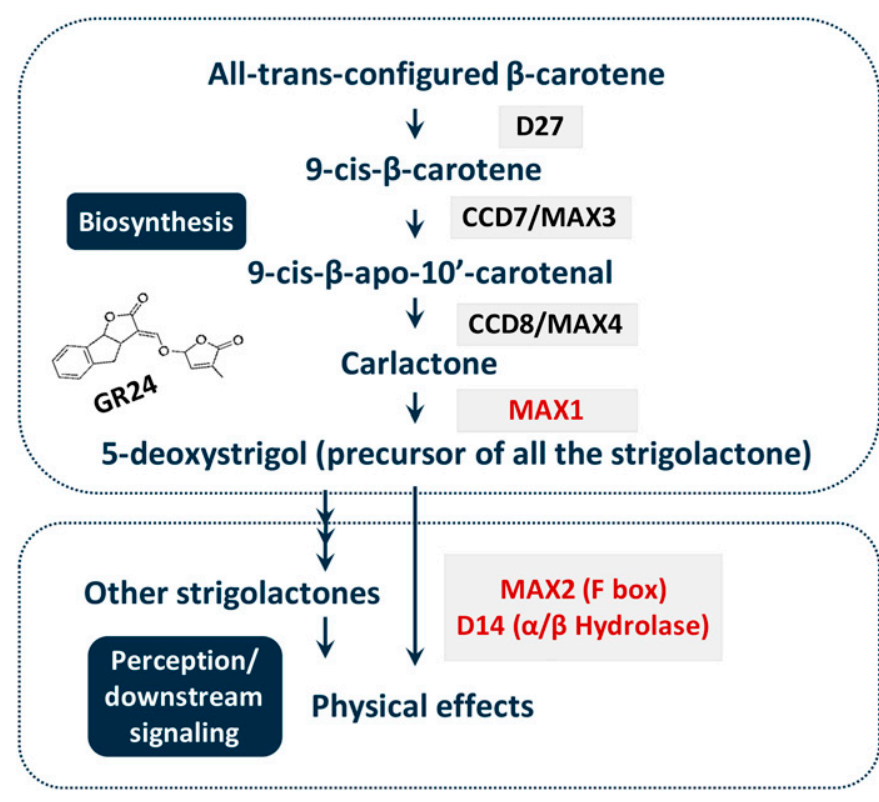

Fig. 1. Genes involved in strigolactone biosynthesis (top) and signaling (bottom). Genes targeted for overexpression constructs (MAX1, MAX2, and D14) are shown in red. 
mixture and incubated at $42^{\circ} \mathrm{C}$ for $1 \mathrm{~h}$. Then, the reaction was terminated by heating at $70^{\circ} \mathrm{C}$ for $15 \mathrm{~min}$. Finally, $2 \mu \mathrm{l}$ of the synthesized cDNA was used as a template to amplify the coding sequence of the three indicated genes using gene-specific primers containing the AscI and BamHI restriction sites following TaKaRa Taq protocol (Supplementary Table S1). The amplified products were digested, purified, and ligated into pG2RNAi2 binary vector using $A s c \mathrm{I}$ and BamHI restriction sites (Supplementary Fig. S1). pG2RNAi2 harbors a green fluorescent protein (GFP) coding gene as reporter for subsequent transgenic hairy root identification. The recombinant binary plasmids were confirmed by Sanger sequencing and introduced to Agrobacterium rhizogenes strain K599 using the freeze-thaw method. Transgenic hairy roots were generated in cultivar Williams 82 according to Kereszt et al. (2007). Transgenic hairy roots expressing the empty pG2RNAi2 binary vector were used as a control, and referred to as WIL82_Rhi. The generated hairy roots were screened using the GFP reporter with the aid of a fluorescence microscope. During the screening process, all nontransgenic roots were removed, leaving only transgenic hairy roots.

Transgenic soybean seedling growth and rhizosphere sample collection. Fresh soil was collected from the East Tennessee Research and Education Center Plant Science Unit just before
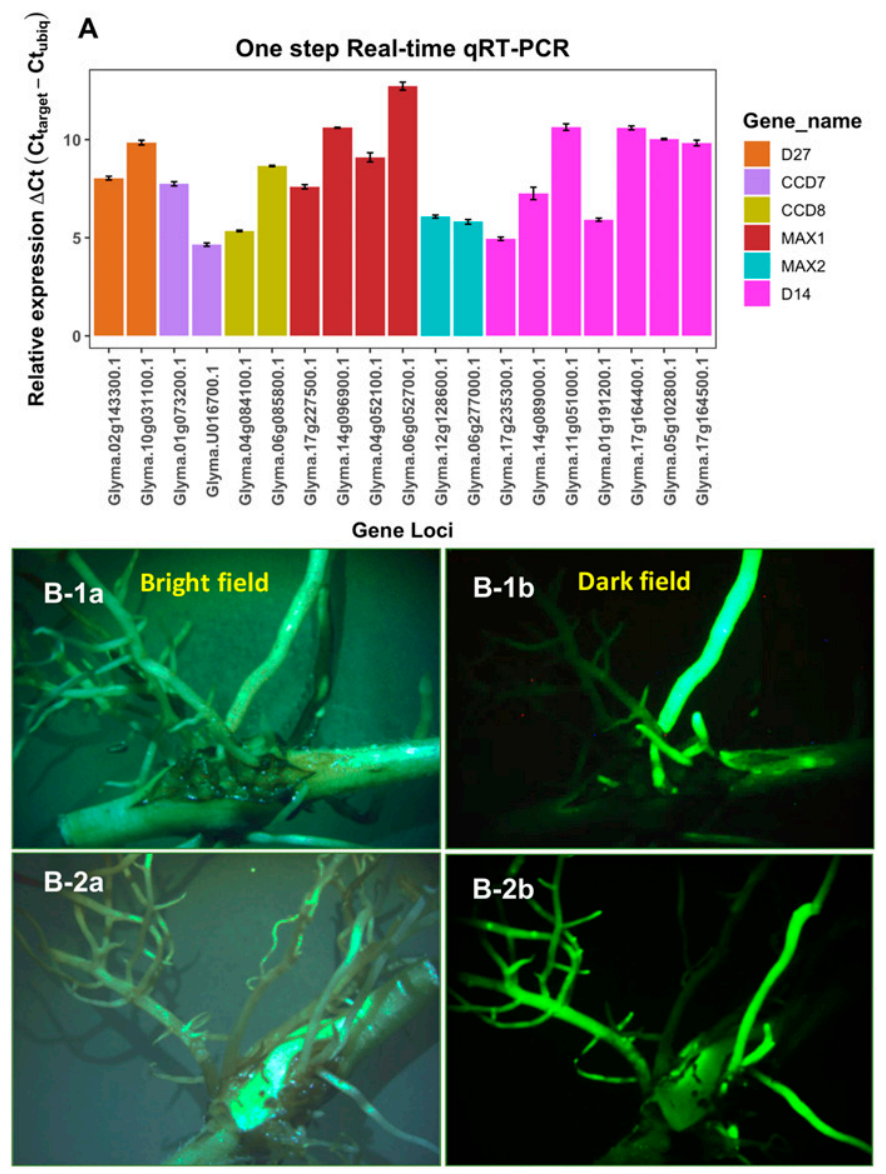

Fig. 2. Strigolactone candidate gene expression and confirmation of overexpression in transformed roots. A, Relative expression across 19 homologs involved in the strigolactone biosynthesis and signaling pathways was assayed with quantitative reverse-transcription (qRT)PCR, with ubiquitin as the constitutive reference gene. B, Green fluorescent protein (GFP) expression in soybean transgenic hairy roots after transformation with Agrobacterium rhizogenes K599 was visualized with a fluorescent microscope. The same roots are visualized under visible light (left) and GFP-specific filter (right). transgenic hairy root screening. After field collection, the soil was immediately transported to the greenhouse. After removing roots and debris, the soil was well homogenized and allocated to pots (diameter of $20 \mathrm{~cm}$ and height of $25 \mathrm{~cm}$ ). Once the transgenic screening was completed, composite plants with similar numbers of transgenic hairy roots were transplanted into the pots and grown in the greenhouse for approximately 1 month until the flowering stage $\left(16 \mathrm{~h}\right.$ of light and $8 \mathrm{~h}$ of darkness at 30 and $20^{\circ} \mathrm{C}$, respectively, with a relative humidity of 60 to $80 \%$ ). Pots with soil but no plants were also maintained in the same conditions, and the soil from these is hereafter referred to as bulk soil. All treatments (MAX1d,D14, MAX2a, WIL82, and bulk soil) were started with 10 biological replicates. All soybean plants and nonplant bulk soils were watered as needed, approximately every other day.

At the flowering stage, soybean rhizosphere soil samples were collected according to Lundberg et al. (2012). Briefly, pots with soybean plants were put upside down into a surface-sterilized metal tray, and soybean roots were gently separated from the soil block. Loosely attached soil was shaken off the soybean roots, and the roots with adherent soil were placed into phosphate buffer (per liter: $6.33 \mathrm{~g}$

\section{Bacteria community CAP plot within soybean rhizosphere}
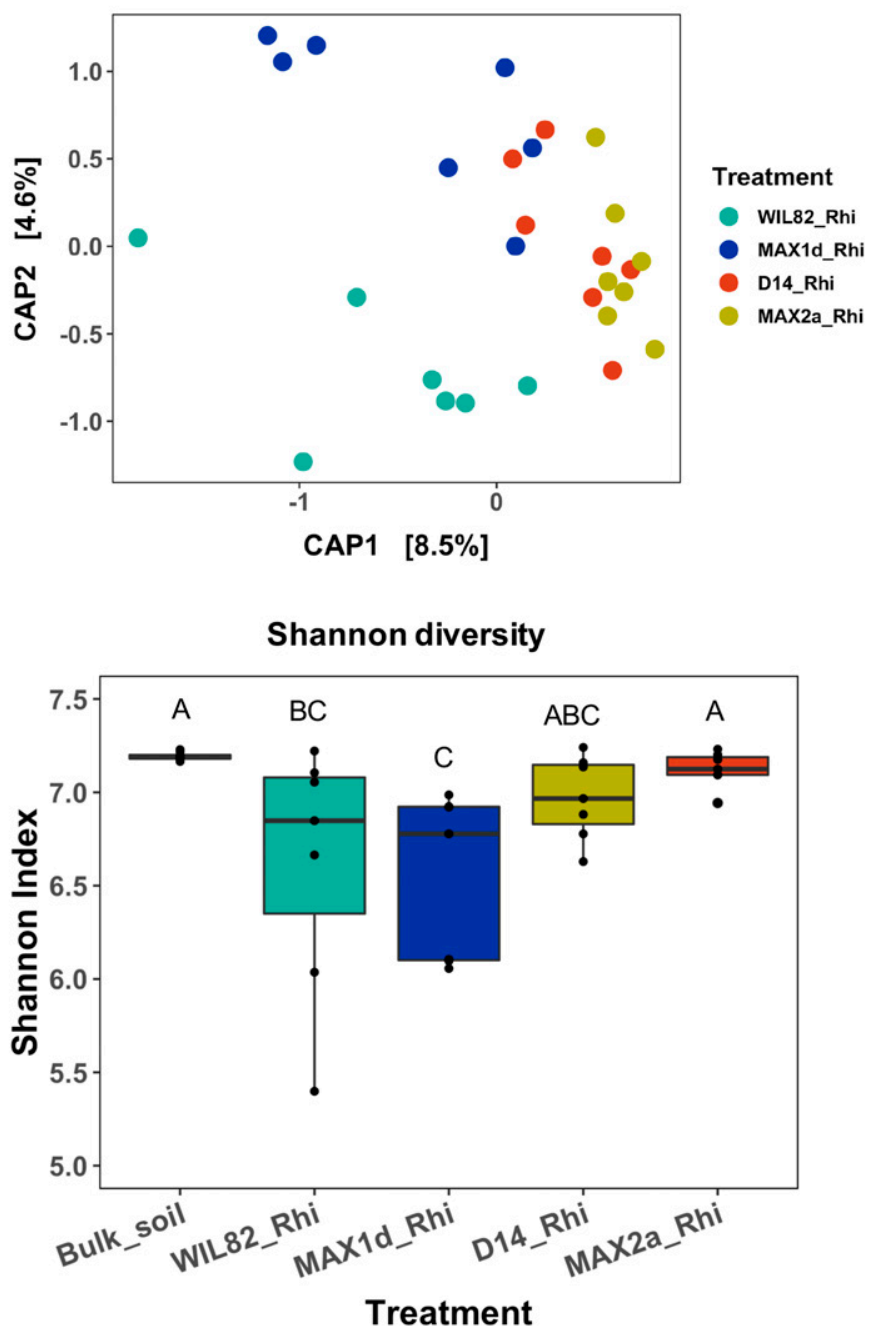

Fig. 3. Differences between treatments in the bacterial community. A, Constrained analysis of principal coordinate analysis (CAP) plot for microbial communities between treatments. B, Shannon diversity box plot for bacterial community. Statistically different groups are indicated by capital letters. 
of $\mathrm{NaH}_{2} \mathrm{PO}_{4} \cdot \mathrm{H}_{2} \mathrm{O}, 16.5 \mathrm{~g}$ of $\mathrm{Na}_{2} \mathrm{HPO}_{4} \cdot 7 \mathrm{H}_{2} \mathrm{O}$, and $200 \mu \mathrm{l}$ of Silwet $\mathrm{L}-77)$. The tubes were vortexed at maximum speed for $30 \mathrm{~s}$ and the slurry was filtered through a $100-\mu \mathrm{m}$ cell strainer. The soil pellet centrifuged from the slurry was collected as the rhizosphere soil sample. A similar amount of bulk soil was collected from the pots with no plant, and processed in parallel with the rhizosphere samples. All of the extracted soil samples were flash-frozen in liquid nitrogen and stored at $-80^{\circ} \mathrm{C}$ before DNA extraction.

DNA extraction, library preparation, and sequencing. Soil DNA was extracted using the PowerSoil DNA isolation kit (Qiagen) following the manufacturer's protocol. We performed $16 \mathrm{~S}$ ribosomal RNA (rRNA) gene-based bacteria profiling using Illumina Miseq 275-bp paired-end sequencing, targeting the V3-V4 region with forward primer $341 \mathrm{~F}=5^{\prime}$-CCTACGGGNGGCWGCAG-3' and reverse primer 785R $=5^{\prime}$-GACTACHVGGGTATCTAATCC-3' (Takahashi et al. 2014). The libraries were prepared following the Illumina $16 \mathrm{~S}$ metagenomic sequencing protocol. Briefly, for the first- step PCR, a 16S rRNA gene-specific primer with adapter overhangs (compatible with Nextera XT index) was used to amplify the $16 \mathrm{~S}$ rRNA V3-V4 region with $2 \times$ KAPA HiFi HotStart ReadyMix with the following PCR cycle: $95^{\circ} \mathrm{C}$ for $3 \mathrm{~min} ; 25$ cycles of $95^{\circ} \mathrm{C}$ for $30 \mathrm{~s}$, $78^{\circ} \mathrm{C}$ for $10 \mathrm{~s}, 55^{\circ} \mathrm{C}$ for $30 \mathrm{~s}$, and $72^{\circ} \mathrm{C}$ for $30 \mathrm{~s}$; and $72^{\circ} \mathrm{C}$ for $5 \mathrm{~min}$. The PCR products were then purified with AMPure XP beads. During the second step of PCR, Illumina dual indices were ligated to the PCR product from step one using the Nextera XT Index Kit with PCR cycle of $95^{\circ} \mathrm{C}$ for $3 \mathrm{~min} ; 8$ cycles of $95^{\circ} \mathrm{C}$ for $30 \mathrm{~s}, 78^{\circ} \mathrm{C}$ for $10 \mathrm{~s}$, $55^{\circ} \mathrm{C}$ for $30 \mathrm{~s}$, and $72^{\circ} \mathrm{C}$ for $30 \mathrm{~s}$; and $72^{\circ} \mathrm{C}$ for $5 \mathrm{~min}$. To minimize the amplification of plant material (chloroplast and mitochondria), peptide nucleic acid (PNA) blockers were used to block mitochondrial and plastid DNA elongation during the first step of PCR (Lundberg et al. 2013): antimitochondrial PNA (mPNA) 5'GGCAAGTGTTCTTCGGA-3' and antiplastid PNA (pPNA) 5'GGCTCAACCCTGGACAG- $3^{\prime}$. Fungal profiling used the internal transcribed spacer (ITS)2 region sequenced on an Illumina MiSeq

\section{Bacteria differential abundance analysis - LefSe results}

A

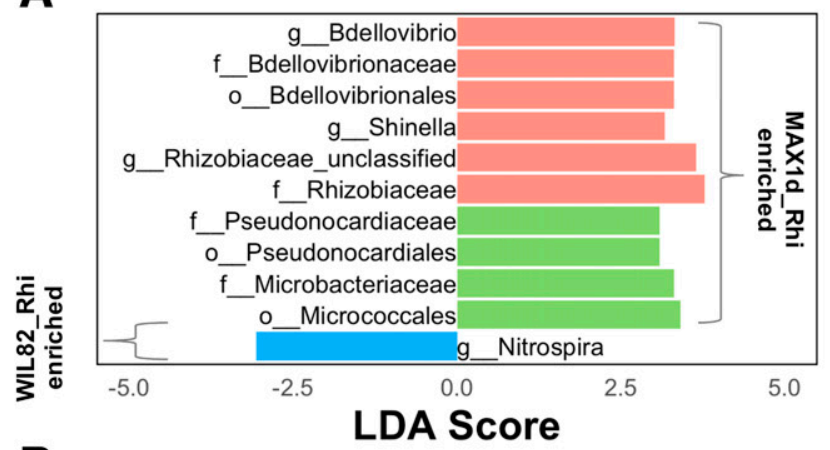

B

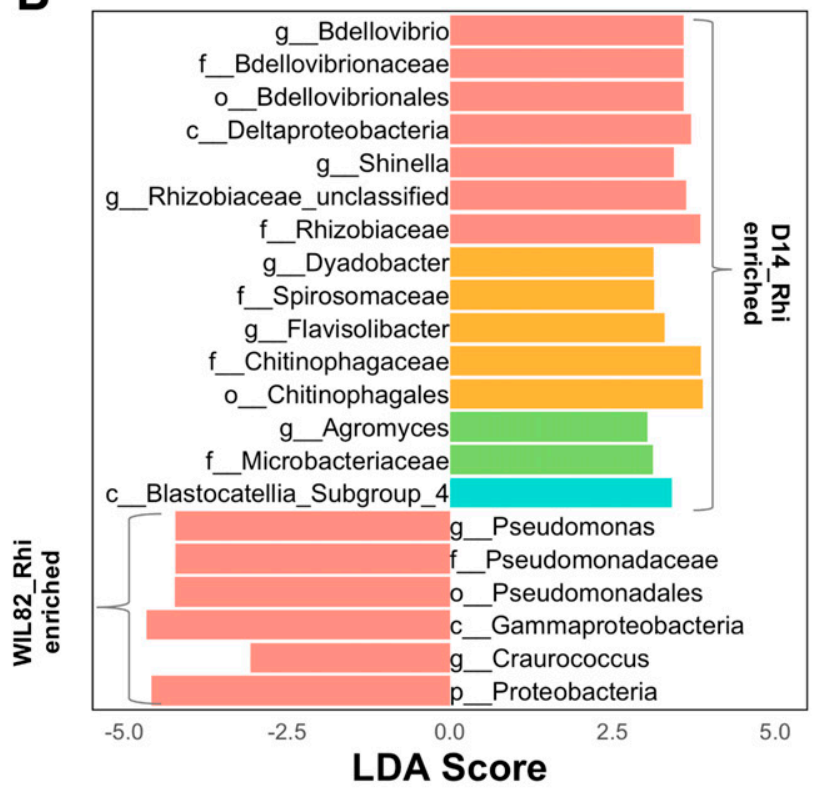

C

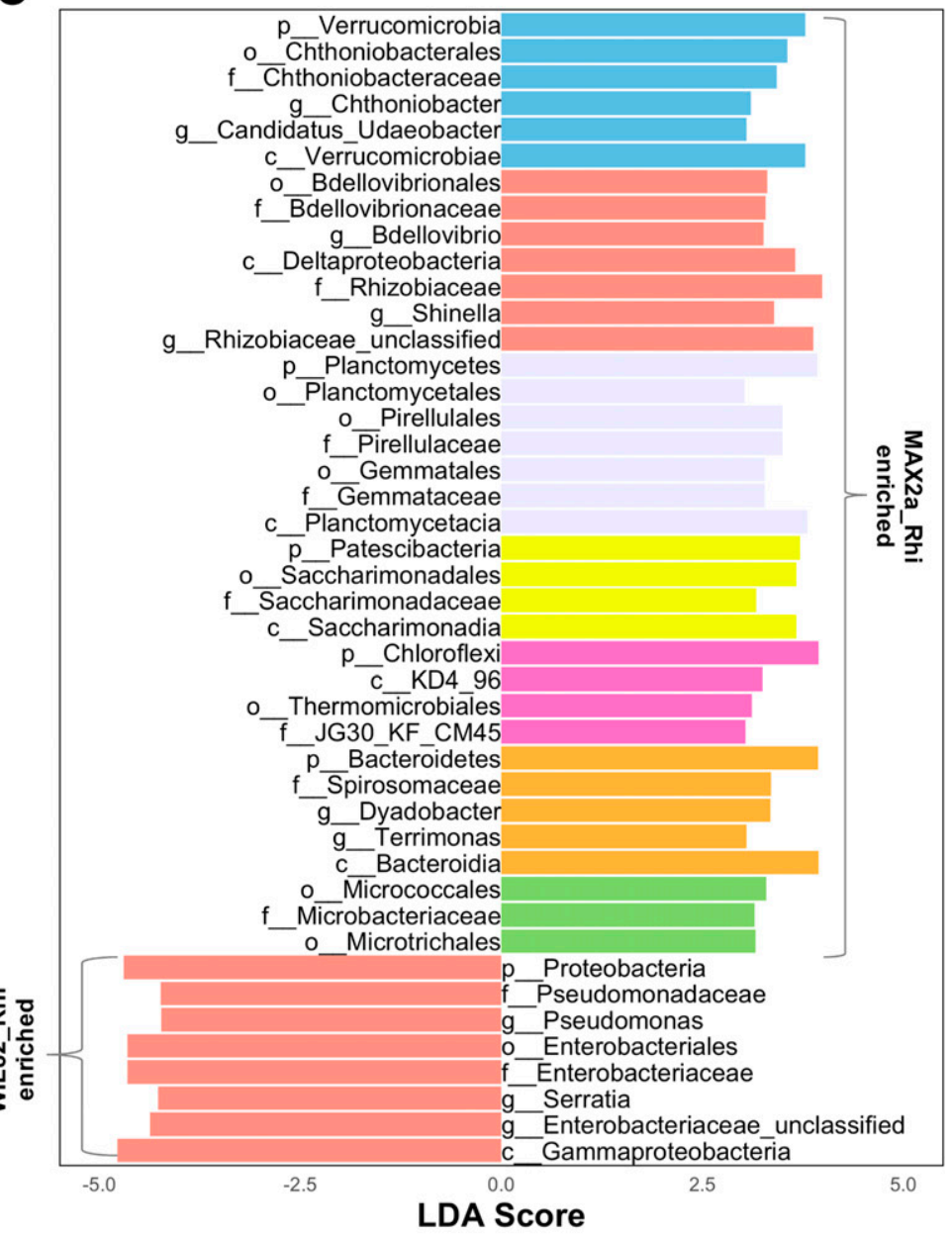

p_Chloroflexi

p_Patescibacteria

p_Planctomycetes
p_Acidobacteria

p_Nitrospirae
p__Verrucomicrobia

Fig. 4. Bacteria differential abundance analysis between overexpression constructs and wild-type rhizospheres. The differential abundance analysis was conducted using LefSe software across all taxonomic levels. All of the listed taxa were either significantly enriched or depleted in the treatments versus the control: A, MAX1d; B, D14; and C, MAX2a. Linear discriminant analysis (LDA) score estimated the effect size. 
with 250-bp paired-end sequencing using six forward and two reverse primers at an equal concentration (Cregger et al. 2018), which are designed to allow better detection of species of Chytridiomycota, Sebacinales, Glomeromycota, Sordariales, Stramenopila, and Archaearhizomycetes. Library preparation also followed the Illumina $16 \mathrm{~S}$ metagenomic sequencing library preparation protocol. The sequencing data were submitted to the SRA database under accession number PRJNA591628.

Sequence analysis. Libraries with very low sequencing yield were removed, leaving seven samples from MAX1d overexpression plants (MAX1d_Rhi), seven samples from D14 overexpression plants (D14_Rhi), seven samples from MAX2a overexpression plants (MAX2a_Rhi), seven samples from plants transformed with a marker gene only (WIL82_Rhi), and eight samples from pots with no plant (bulk soil). Mothur software was used to process $16 \mathrm{~S}$ rRNA gene and ITS2 region sequences (Schloss et al. 2009). For 16S rRNA gene sequences, operational taxonomic units (OTUs) were clustered at $97 \%$ similarity using aligned sequences and classified against the SILVA 132 taxonomy reference database using a naive Bayesian classifier (Kozich et al. 2013; Wang et al. 2007). Because sequence variation within the $16 \mathrm{~S}$ rRNA V3-V4 region is not sufficient to distinguish non-A. rhizobium with our vector carrier (A. rhizogenes $\mathrm{k} 599$ ), any sequences classified as A. rhizobium were removed from downward OTU clustering and community analysis. Detailed exploration of this OTU as well as qPCR-based quantification is available in Supplementary File S1. For ITS2 sequences, OTUs were clustered at $97 \%$ similarity using unaligned sequences, which were taxonomically classified based on the UNITE v8 reference (Nilsson et al. 2019). For both 16S and ITS2, any sequence pairs (forward and reverse reads) with a mismatch within the primer region were removed before assembly. Chimera sequences were detected and discarded using the vsearch tool based on the UCHIME algorithm (Edgar et al. 2011; Rognes et al. 2016). Sequences that belonged to chloroplast, mitochondria, eukaryotes, and archaea were discarded before OTU clustering for $16 \mathrm{~S}$ rRNA data. Similarly, nonfungal sequences, including unknown, Alveolata, Amoebozoa, Apusozoa, Choanoflagellozoa, Chromista, Cryptista, Euglenozoa, Eukaryota, Incertae_sedis, Filasteriae, Glaucocystoplantae, Haptista, Heterolobosa, Ichthyosporia, Metazoa, Picozoa, Planomonada, Protista, Protozoa, Rhizaria, Rhodoplantae, Stramenopila, and Viridiplantae species, were removed before OTU clustering and classification. To alleviate the bias introduced by uneven sequencing depth, rarefaction at the minimum sequencing depth of 26,012 (16S rRNA gene) and 9,169 (ITS2) was used for normalization. OTUs represented by a single read were removed before subsequent microbial community analysis in $\mathrm{R}$.

To compare the compositional differences of bacterial and fungal communities between treatments, $\beta$ diversity between samples was calculated based on Bray-Curtis dissimilarity and visualized by principal coordinates analysis (PCoA) and constrained analysis of PCoA (CAP) plots. Shannon $\alpha$ diversity metrics were compared between treatments using a Kruskal-Wallis test, followed by pairwise comparison using Dunnett's test at the threshold $P$ value < 0.05 . Differential abundance of bacteria and fungi at individual taxa level was analyzed using LefSe (Segata et al. 2011). Tax4Fun2 was used to infer the functional capacity of bacterial communities based on taxonomic information (Wemheuer et al. 2018). The metabolic pathway profiles generated from Tax4Fun2 were used as input for DESeq2 to test the treatment effects on individual metabolic pathways (Love et al. 2014). KEGG pathways with differential abundance between treatments were extracted for visualization using pheatmap (Kolde 2015). Similarly, FUNGuild was used to summarize fungal functions and guilds between treatments
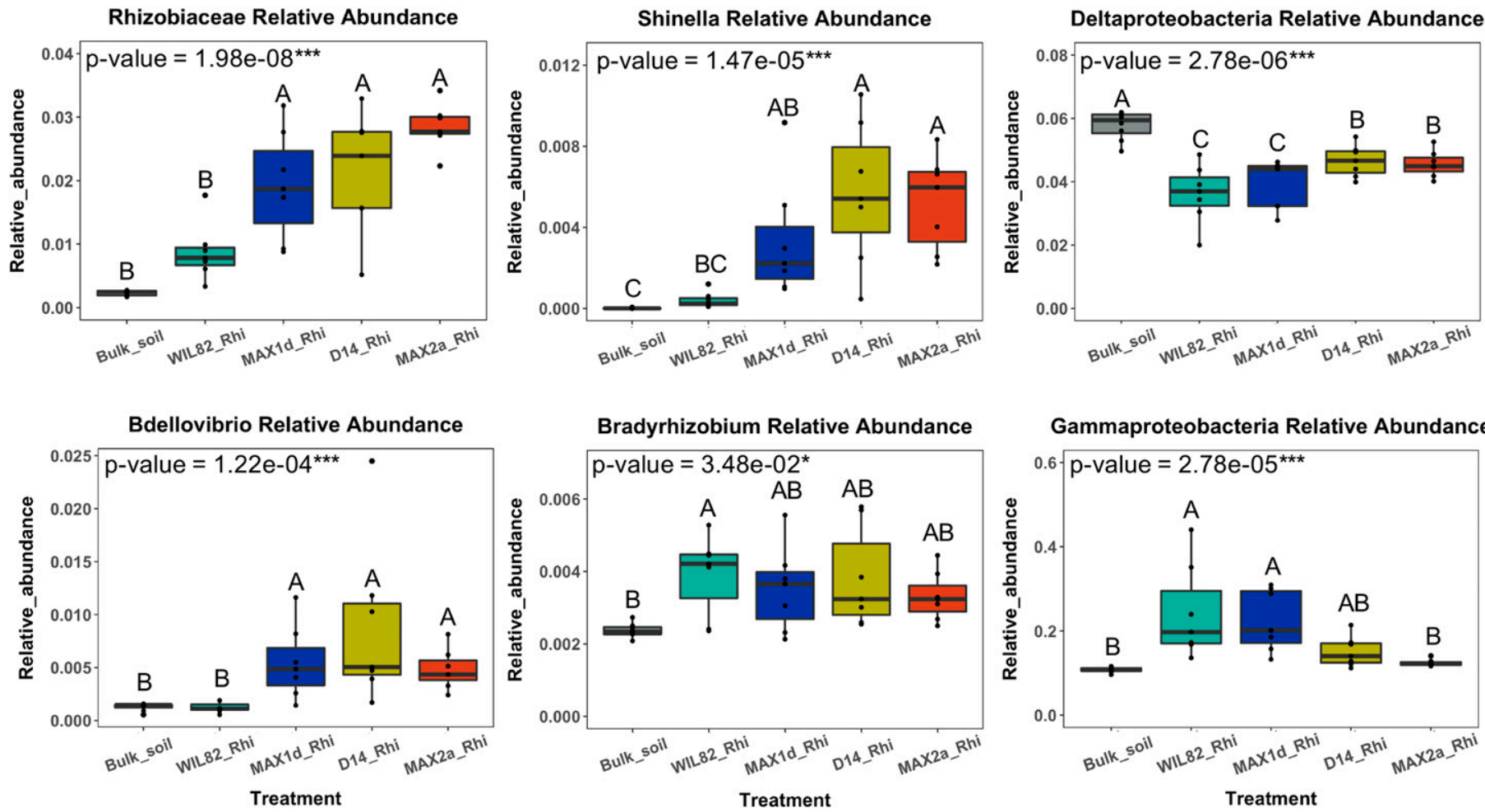

Fig. 5. Relative abundance bar plots for bacterial taxa between treatments. The $P$ value was calculated based either on one-way analysis of variance or nonparametric Kruskal-Wallis test, depending on the normality of the data. Capital letters at the top of each bar represent the differences between treatments based on a pairwise comparison based either on Tukey's honestly significant difference or Dunn's test. 
(Nguyen et al. 2016). The relative abundance of predicted fungi guilds was compared between treatments using either one-way analysis of variance or a Kruskal-Wallis test, depending on the distribution of corresponding dependent variables.

\section{RESULTS}

Generation of transgenic soybean hairy root plants. To select candidate genes for transformation, we targeted highly expressed genes from the most downstream steps of the strigolactone biosynthesis and perception pathways (Ruyter-Spira et al. 2013; Xie et al. 2010) (Fig. 1). qRT-PCR results of 19 strigolactone-associated genes detected expression in root tissue for all 19 homologs (Fig. 2A). The most highly expressed homologs of MAX1, D14, and MAX2 genes were selected (i.e., Glyma.14g096900, MAX1d; Glyma.17g235300, D14; and Glyma.12g128600, MAX2a) as targets for generating overexpression constructs for producing transgenic soybean hairy roots in the Williams 82 genotype. Transgenic hairy root plants expressing the empty pG2RNAi2 binary vector with the GFP reporter were used as control. Microscopic screening results indicated that the overexpression constructs were successfully transferred and expressed in the selected hairy roots of all individuals (Fig. 2B).

Microbial community composition and structure in the soybean rhizosphere. In total, 1,810,039 16S rRNA gene and 971,362 ITS2 amplicon sequences were retained after initial trimming and screening with Mothur software (Schloss et al. 2009). These sequences were clustered into 42,313 bacterial OTUs and 4,634 fungal OTUs across 36 samples (7 MAX1d_Rhi, 7 D14_Rhi, 7 MAX2a_Rhi, 7 WIL82_Rhi, and 8 bulk soil). After rarefaction and removal of singletons, 12,368 bacterial OTUs and 1,607 fungal OTUs remained, which were classified into 1,048 genera and 750 species, respectively (Supplementary Table S2). Rarefaction plots indicated consistent sampling of taxa across individual libraries and sufficient sequencing depth for the fungal community; however, species richness was likely not fully sampled for the bacterial community (Supplementary Fig. S2). The soybean rhizosphere was different in microbial composition from the bulk soil (Supplementary File S1), mirroring previous studies of the rhizosphere effect in soybean (Liu et al. 2019; Mendes et al. 2014; Zhang et al. 2018).

Rhizosphere bacterial composition and diversity across treatments. The influence of strigolactone-associated gene overexpression on soybean rhizosphere bacterial composition was small but significant. The constrained ordination explained $16.37 \%$ of the total variation between samples (excluding bulk soil), and the CAP plot showed the clear separation of soybean control (WIL82_Rhi) and overexpression (MAX1d_Rhi, D14_Rhi, and MAX2a_Rhi) samples (Capscale, $\mathrm{F}_{(3,24)}=1.56, P=0.007$ ) (Fig. 3A). The composition of the bacterial community also differs between the individual overexpression gene treatments (Capscale, $\mathrm{F}_{(2,18)}=1.49$, $P=0.028)$. Rhizosphere bacterial communities from the plants overexpressing the strigolactone perception genes $D 14$ and MAX2a were very similar to each other and significantly diverged in taxonomic composition from the rhizosphere communities of plants overexpressing MAXld, a strigolactone biosynthesis gene (Capscale, $\left.\mathrm{F}_{(1,19)}=1.95, P=0.007\right)$. Differences in Shannon $\alpha$ diversity of the bacterial community were less evident (Fig. 3B). Of the three genes, MAX2a_Rhi was the only one significantly different from the wild-type WIL82_Rhi with higher overall $\alpha$ diversity. MAX2a_Rhi was also significantly more diverse than MAX1d_Rhi.

By examining each treatment against the soybean control, we found specific enriched and depleted bacterial taxa: MAX1d had 10 enriched and 1 depleted, D14 had 15 enriched and 6 depleted, and
MAX2a had 36 enriched and 8 depleted (Fig. 4; Supplementary Table S3). The families Microbacteriaceae, Rhizobiaceae (especially genus Shinella), and Bdellovibrionaceae (especially genus Bdellovibrio) were consistently enriched across overexpressing roots compared with the soybean empty-vector control roots (Fig. 5). A few enriched and depleted taxa were shared by more than one treatment. For example, Deltaproteobacteria and Dyadobacter were significantly enriched in D14- and MAX2a-overexpressing roots compared with control roots while Gammaproteobacteria (Pseudomonas specifically) was significantly depleted. However, none of these three changes were detectable for the MAXIdoverexpressing roots.

Rhizosphere fungal composition and diversity across treatments. In contrast to bacterial communities, the differences in fungal community composition between control soybean roots and overexpressing roots was not significant at a $P$ value cutoff of 0.05 (Capscale, $\mathrm{F}_{(3,24)}=1.22, P=0.098$ ) (Fig. 6), although the constrained ordination explained $13.23 \%$ of overall variation (excluding bulk soil). However, the low $P$ value of 0.098 indicates that more biological replicates or sequencing depth may be needed to decisively determine whether differences are present. Nor did we detect any significant differences between MAX1d-, D14-, and

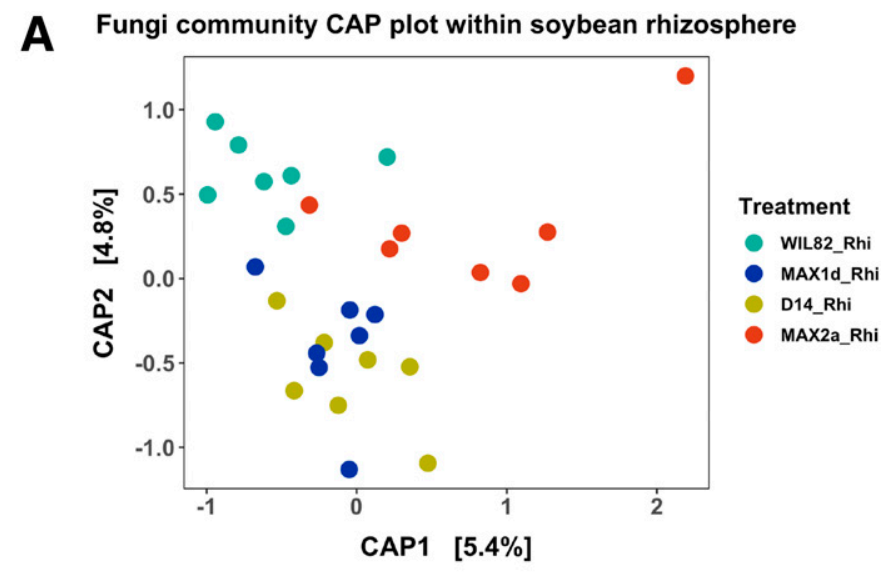

B Shannon diversity

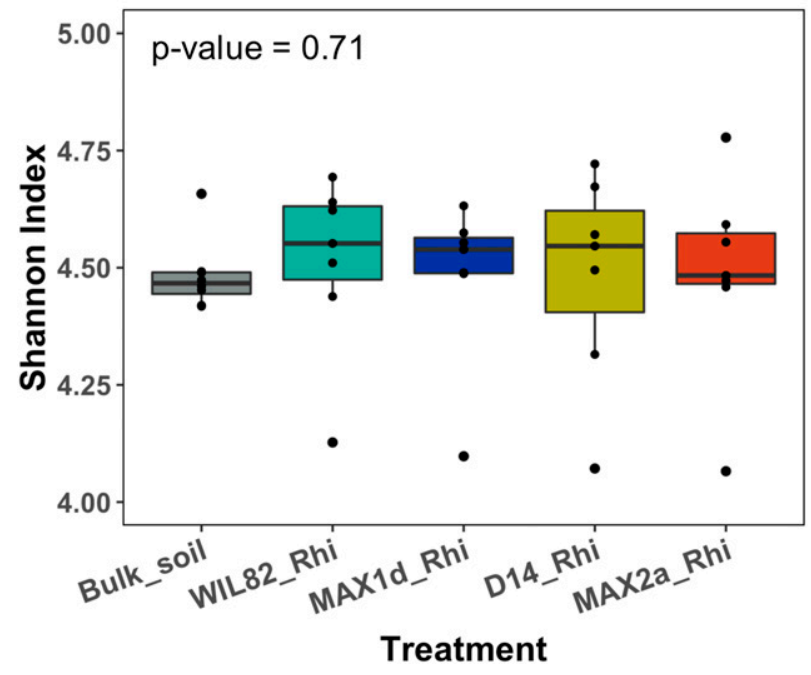

Fig. 6. Differences between treatments in the fungal community. A, Constrained analysis of principal coordinate analysis (CAP) plot for microbial communities between treatments. B, Shannon diversity box dot plot for fungal community. No statistically different groups were detected. 
MAX2a-overexpressing roots (Capscale, $\mathrm{F}_{(2,18)}=1.07, P=0.306$ ). In terms of fungal community diversity, the Shannon $\alpha$ diversity was similar between the bulk soil controls and soybean rhizosphere as well as between the soybean control roots and overexpression roots (Kruskal-Wallis $\chi^{2}=6.53, P=0.16$ ) (Fig. 6B).

Despite the lack of significant differences in overall community composition, individual taxa were significantly enriched or depleted by the overexpression of strigolactone biosynthesis and perception genes (Fig. 7; Supplementary Table S3). Specifically, Fusarium solani was consistently enriched in the soybean rhizosphere with the overexpression of MAXId and D14 when compared with the soybean control (Fig. 8). The Rhizophlyctidales order was selectively enriched in MAX2a-overexpressing roots compared with soybean control, while Spizellomycetales and Sordariales orders were less abundant compared with soybean control soybean. Surprisingly, the symbiotic arbuscular fungal family Glomeraceae was not significantly different in abundance between soybean control and all overexpression treatments.

Predicted functional differences between treatments. Although Tax4Fun functional prediction suggested distinct functional capacities between bulk soil and the soybean rhizosphere bacterial communities, there were no significant differences predicted between the soybean control and overexpression treatments (Fig. 9A). FUNGuild was applied to link fungal taxa information with fungi ecological guilds. The trophic modes composition of the fungal communities differed only slightly between bulk soil controls and rhizosphere as well as among overexpression treatments. Statistically, saprotrophs were significantly decreased in $M A X 2 a$-overexpressing roots and symbiotrophs were significantly reduced in MAX1d- and D14-overexpressing roots in comparison with the soybean control treatment (Fig. 9B).

\section{DISCUSSION}

The rhizosphere is one of the most dynamic interfaces between the soil and plant roots, with a specialized microbial community that influences plant activities (Philippot et al. 2013). In this study, we modified the expression of soybean (Williams 82) strigolactone biosynthesis and its perception genes using the hairy root transformation approach (Kereszt et al. 2007) and investigated the resulting differences in soybean rhizosphere bacteria and fungi communities. Our initial results indicate that altered expression of

\section{Fungi differential abundance analysis - LefSe results}

A
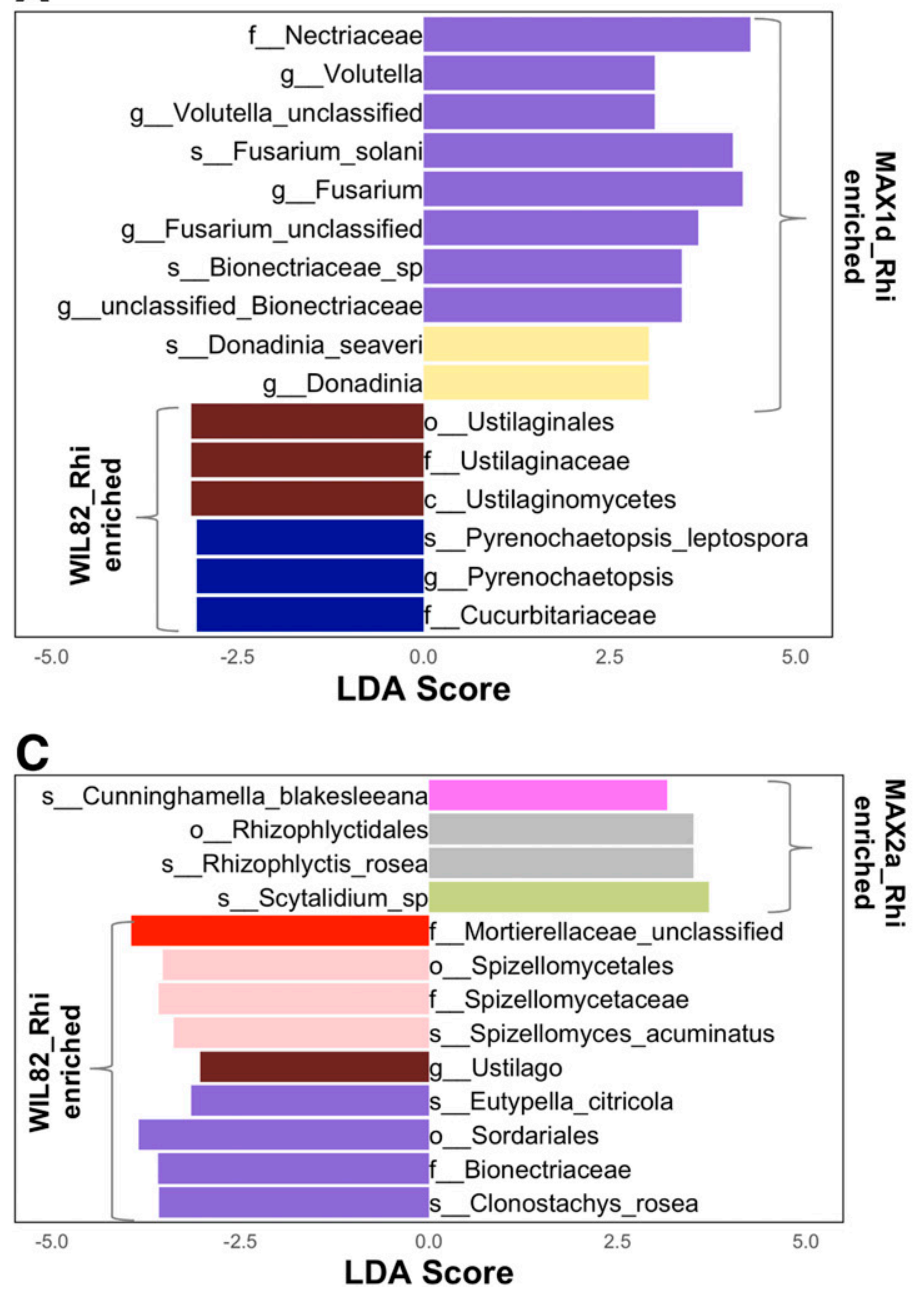

B

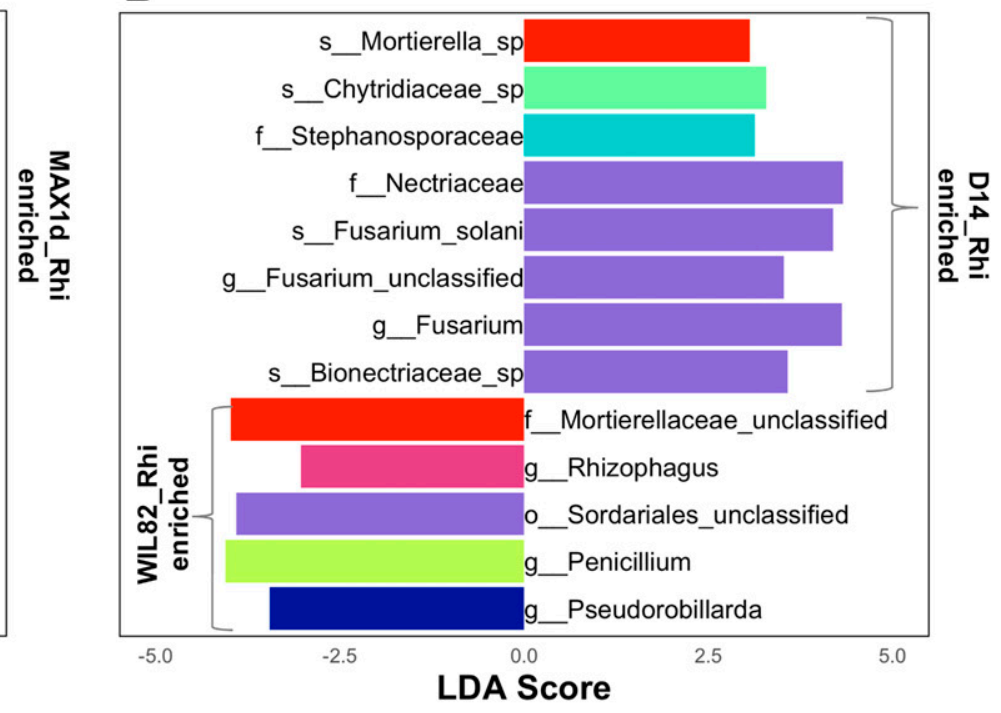

Fig. 7. Fungi differential abundance analysis between overexpression constructs and wild-type rhizospheres. Differential abundance analysis was conducted using LefSe software across all taxonomic levels. All of the listed taxa were either significantly enriched or depleted in the treatments versus the control: A, MAX1d; B, D14; and C, MAX2a. Linear discriminant analysis (LDA) score estimated the effect size. 
strigolactone-related genes influences bacterial community assembly in the rhizosphere of soybean, warranting future examination of the role of strigolactone as an important signaling compound in shaping the plant microbiome.

Because strigolactones have multifaceted functions both in planta and ex planta (López-Ráez et al. 2017), the alterations of rhizosphere bacterial and fungal taxa abundance identified here could have been elicited directly or indirectly. Strigolactones act as a direct signaling molecule after exudation into the rhizosphere for some AMF species (Akiyama et al. 2005; Besserer et al. 2006) and parasitic plants (Cook et al. 1966) but their influence on other taxa is not yet well understood. Alternatively, strigolactones could be utilized by bacteria as a specific preferred carbon source. However, strigolactones are exuded at the nano- and picomolar quantities outside of the plant and tend to decompose rapidly (Xie et al. 2010), making their primary impact as a carbon source less likely.

The differences in microbial communities could also be from secondary effects of the strigolactone gene expression alterations. Strigolactones act as signaling compounds within the plant, and they are directly involved in both root morphology and hormone cross-talk in many plant species (Waters et al. 2017). Haq et al. (2017) generated GmMAX3b overexpression and knockdown lines of soybean using the same type of hairy root system as the experiments reported here. Overexpression increased nodulation while knockdown of expression decreased nodulation. Also, endogenous hormone levels were influenced: GmMAX3b overexpression yielded reduced abscisic acid (ABA) and increased jasmonic acid (JA) while the knockdown yielded the opposite trends. This was also reflected in large-scale gene expression changes for both overexpression and knockdown lines, including altered expression of more than 2,000 genes. Rehman et al. (2018) knocked down two other MAX genes, GmMAXla and GmMAX4a, in soybean also using a hairy root transgenic system and found decreased nodulation in both lines. They also identified different endogenous hormone levels: increased ABA and JA as well as decreased auxin. It is still an open question as to whether these alterations in other hormone levels and gene expression patterns could yield different root exudation profiles for compounds other than strigolactones, emphasizing the need for root exudate profiling in future work.

The overexpression of strigolactone-associated genes modified soybean rhizosphere bacterial community. In this study, the overexpression of strigolactone biosynthesis and signaling genes in soybean roots had significant impacts on rhizosphere bacteria assembly, with distinct bacterial community compositions between the MAX1d-, D14-, and MAX2a-overexpressing roots and the control Williams 82 roots. The bacterial composition of MAX2a and $D 14$, both genes involved in strigolactone perception, were quite similar to each other and differed from MAX1d, a gene involved in strigolactone biosynthesis. It is worth noting that $M A X 2 a$ participates in both strigolactones and karrikin (KAR) signaling pathways, with the latter pathway being revealed to trigger seed germination after fires or burning events (Flematti et al. 2009; Nelson et al. 2011; Smith and Li 2014). The distinct differences of rhizosphere bacteria between MAX1d and MAX2a overexpression, which is less evident between MAX1d and D14 overexpression, may reflect the dual role of the MAX2a gene in both strigolactones and KAR signaling pathways (Nelson et al. 2011). Whether the microbial community differences found here were influenced by KAR signaling is an open question. Follow-up studies with mutants of the genes studied here and other KAR signaling genes would benefit from directly measuring strigolactone and KAR production and exudation rates to address this.
Despite the taxonomic differences between treatments, we did not observe a significant impact of the overexpression of strigolactone genes on predicted rhizosphere bacterial metabolic pathways. This inconsistency between composition and functional

\section{Fusarium solani Relative Abundance}

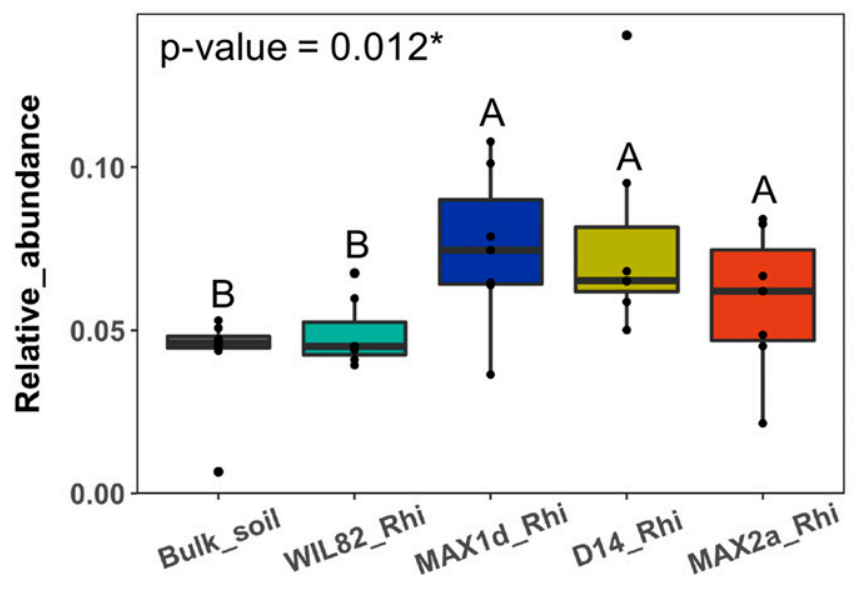

Glomeraceae family Relative Abundance

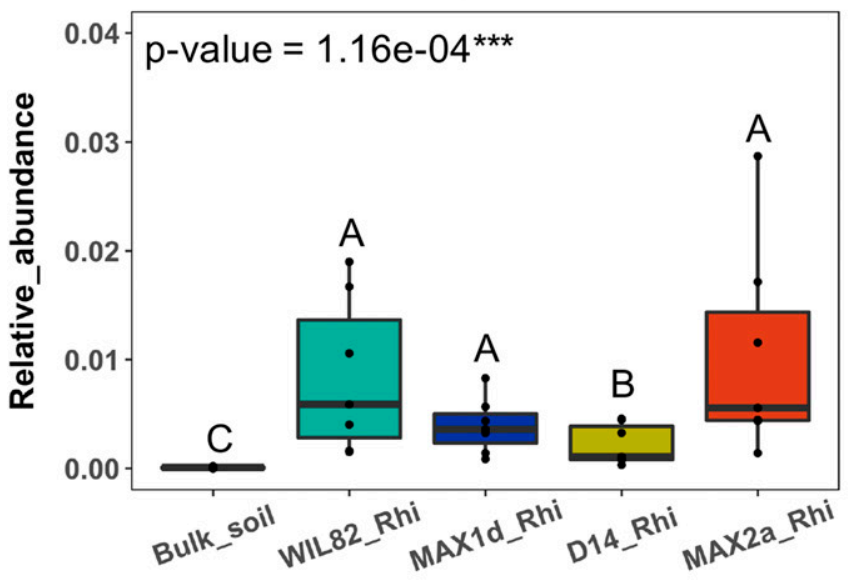

Nectriaceae family Relative Abundance

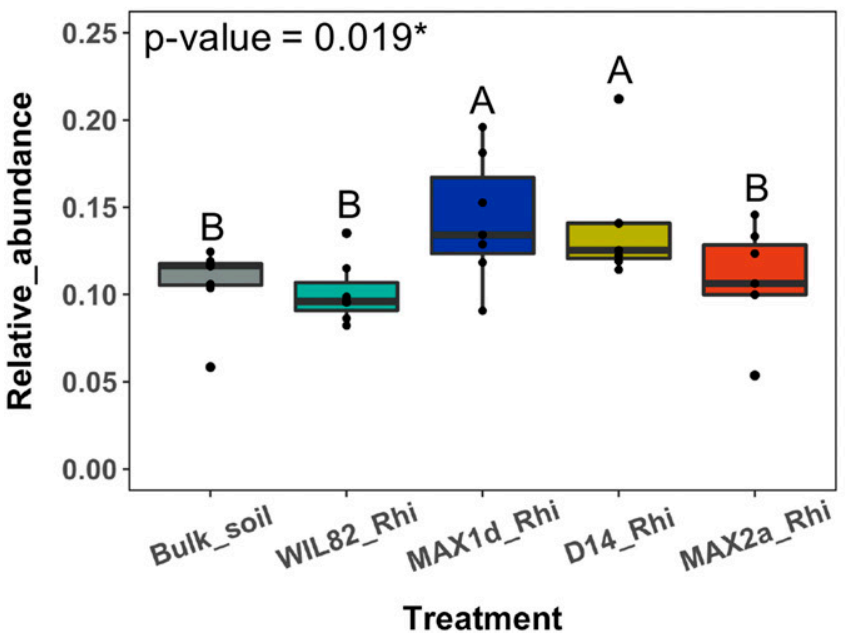

Fig. 8. Relative abundance bar plots for fungal taxa between treatments. The $P$ value was calculated based either on one-way analysis of variance or nonparametric Kruskal-Wallis test, depending on the normality of the data. Capital letters at the top of each bar represent the differences between treatments based on a pairwise comparison based either on Tukey's honestly significant difference or Dunn's test. 
response could be explained by the functional redundancy between bacteria taxa. Alternatively, this discrepancy could be caused by a lack of functional annotation for many soil bacterial species.

Recent studies applying gene manipulation and $\mathrm{GR}^{24}$ applications suggest that strigolactones impact legume nodulation (De Cuyper et al. 2015; Foo and Davies 2011; Foo et al. 2013; Haq et al. 2017; Peláez-Vico et al. 2016; Rehman et al. 2018). The differential abundance analysis from this study revealed stronger recruitment of Rhizobiaceae spp. from bulk soil to the soybean rhizosphere in response to overexpression of strigolactone biosynthesis and perception genes, suggesting that strigolactones play a role in the selective recruitment of microbes to the rhizosphere prior to the establishment in nodules. We also detected a dramatically high enrichment of the genus Rhizobium in the rhizosphere of overexpressing roots in comparison with the soybean control roots. However, we were not able to distinguish nitrogen-fixing rhizobia from A. rhizogenes (the delivery agent of the overexpression constructs) due to the insufficient resolution of $16 \mathrm{~S}$ rRNA sequencing. Despite the widely reported increase in nodulation stimulated by higher strigolactone concentrations, we did not detect
A

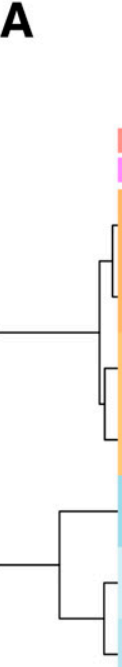

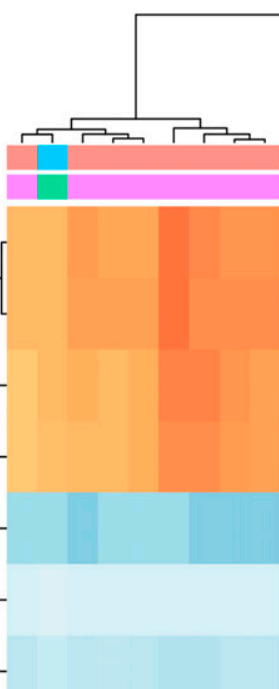

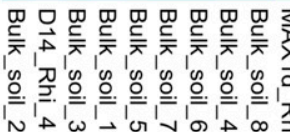

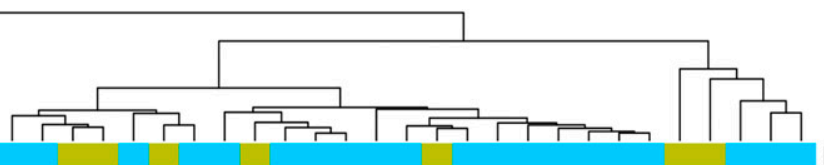
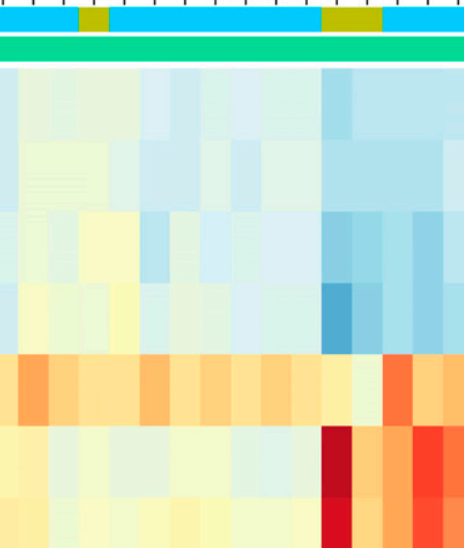

Expression

Compartment

Polycyclic aromatic hydrocarbon degradation

Furfural degradation

Microbial metabolism in diverse environments

Aminobenzoate degradation

Other glycan degradation

Phosphotransferase system (PTS)

Fructose and mannose metabolism

B

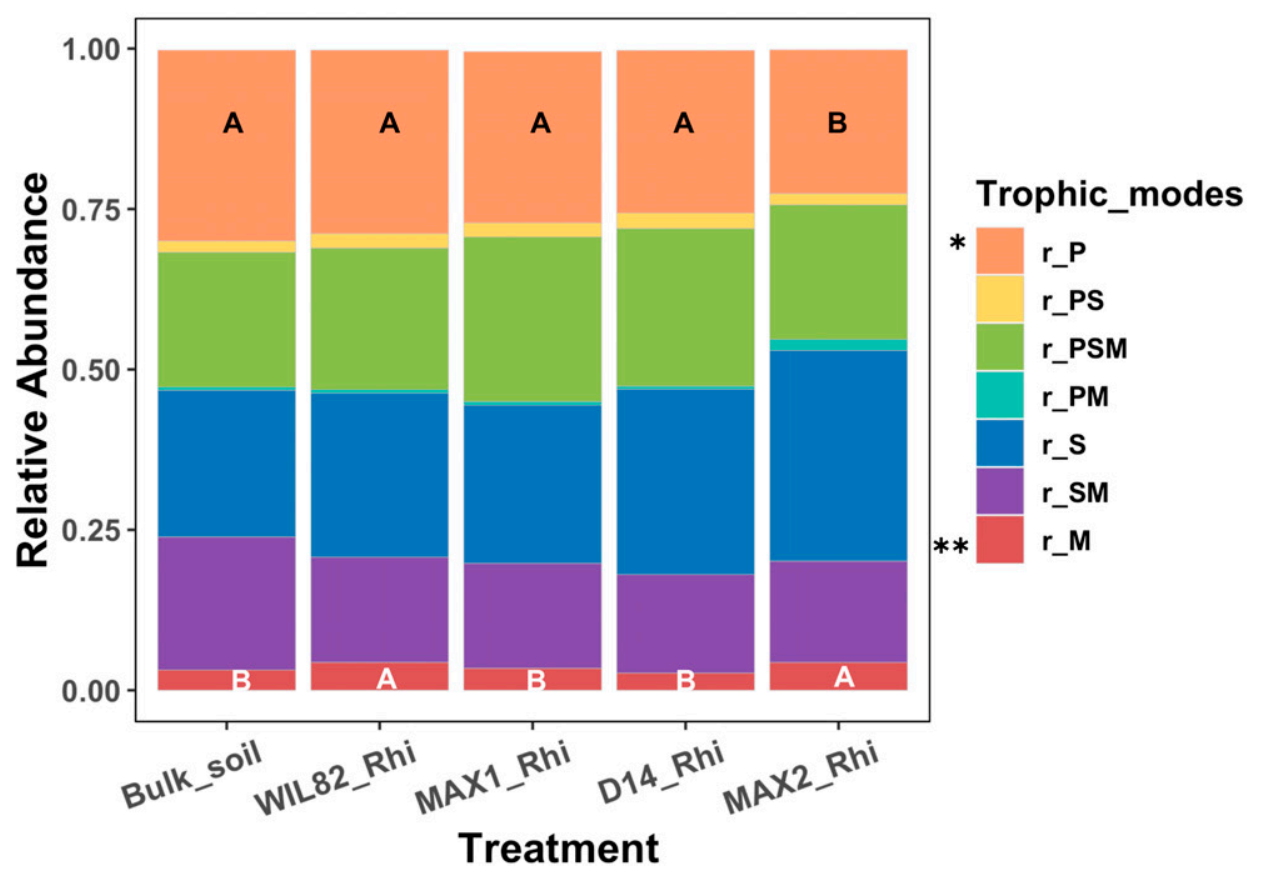

Fig. 9. Predicted functional profiles for bacterial and fungal communities. A, Bacteria metabolic pathway heatmap based on Tax4Fun2 functional annotation; B, Stacked bar plot of fungi trophic modes based on FUNGuild mediated functional annotation. Predicted trophic modes are labeled pathotrophs (P), saprotrophs (S), and symbiotrophs (M). Multitrophic assignments are combinations of those letters (e.g., PS for pathotrophsaprotrophs). 
significant abundance changes between soybean control and overexpressing roots for Bradyrhizobium and Sinorhizobium spp., the most predominant nodule-formatting rhizobia of soybean (Lindström et al. 2010). This is consistent with McAdam et al. (2017), who found that the deficiency of strigolactone biosynthesis gene CCD8 did not change the exudation of flavonoids, which function as a chemoattractant to recruit Bradyrhizobium japonicum (Graham 1991; Khan and Bauer 1988).

Our experiment revealed that the bacteria genera Shinella and Bdellovibrio were significantly enriched in the soybean rhizosphere across all three overexpressed genes compared with the soybean control line. One Shinella strain, Shinella kummerowiae, was isolated from the herbal legume Kummerowia stipulaceae nodules and found to have nodulation and nitrogen-fixing genes (Lin et al. 2008), while other Shinella spp. have been repeatedly found in contaminated or wastewater functioning as nitrogen-reduction or sulfolaneassimilating bacteria (Mu et al. 2018; Vaz-Moreira et al. 2010). The enrichment of the Shinella genus in response to overexpression of strigolactone could belong to any of the aforementioned strains, suggesting shotgun metagenome sequencing as the next approach for species-level identification. The genus Bdellovibrio includes bacteria able to parasitize and kill other bacteria. Bdellovibrio bacteriovorus has been reported as a predatory bacterium of soybean bacterial blight disease and has been proposed as a potential biocontrol for other phytopathogens (Olanya and Lakshman 2015; Scherff 1973). Our finding is consistent with the study of Nasir et al. (2019), which revealed decreased Bdellovibrio spp. abundance in the rhizosphere of rice mutants defective in strigolactone biosynthesis (CCD7) and signaling (D14) genes compared with wild-type rice (Nasir et al. 2019).

Strigolactone overexpression impacts individual fungal taxa but is insignificant at the community level. After examining the fungi compositional response to strigolactone overexpression, we did not observe significant changes at the community level. This is in contrast to Carvalhais et al. (2019), who disrupted CCD8/MAX4 in Arabidopsis thaliana and found significant modulation of the rhizosphere fungi community (Carvalhais et al. 2019). Strigolactones are widely recognized as a triggering signal for arbuscular mycorrhizal fungi hyphae growth and activities (Akiyama et al. 2005; Besserer et al. 2008), and disrupting gene expression may have a larger community impact than our approach to overexpress genes. FUNGuild predicted $4 \%$ of fungi in the soybean rhizosphere as mycorrhizal. The surprisingly low abundance of mycorrhizal fungi was also reported in a poplar root microbiome study (Cregger et al. 2018). The low abundance of mycorrhizal fungi could be due to some fungal endophytes shifting their ecological strategy from an endophytic to a saprophytic phenotype (Promputtha et al. 2007), a possibility that is supported by a large percent of fungi in the soybean rhizosphere being predicted as pathotroph-symbiotroph.

At the individual taxa level, we found significant and consistent enrichment of $F$. solani species in MAX1d- and D14- but not $M A X 2 a$-overexpressing root rhizospheres. $F$. solani is a species complex that includes a number species characterized as fungal pathogens causing sudden death syndrome, which was ranked as one of the most devastating soybean diseases across the top 10 major soybean-growing areas (Roy 1997; Wrather et al. 2001). Although ITS2 sequencing-based results were not sufficient to determine whether these enriched $F$. solani are pathogenic or not, these results warrants future investigation. There are a number of reports indicating that strigolactones inhibit fungal pathogens based on both gene mutation and $\mathrm{GR}^{24}$ assays (Belmondo et al. 2017; Dor et al. 2011; Torres-Vera et al. 2014). However, other studies have not found impacts of strigolactones on fungal disease development (Blake et al. 2016; Foo et al. 2016).
Conclusion and perspectives. Although the plant hormones strigolactones are known to influence plant-fungus interactions, particularly AMF, we demonstrate that modulating the strigolactone signaling and biosynthesis pathway alters the rhizosphere bacteria community as well. For both fungi and bacteria, we demonstrate microbial alterations in the rhizosphere in terms of community composition, structure, and potential function. Increased occurrence of taxa such as Rhizobiaceae, Shinella, Bdellovibrio, and F. solani indicate that this pathway may be important for signaling of microbes involved in recruiting specific taxa for nodulation and nitrogen fixation as well as influencing microbe-microbe interactions and pathogens. Considering the multifunction feature of strigolactones, a more detailed investigation using stable transgenic lines, with and without functional destruction in AMF or nodule formation together with root exudation profiling, will provide a clearer picture of the direct and indirect impacts exerted by strigolactones.

\section{ACKNOWLEDGMENTS}

We thank Lori Osburn for her help in managing the greenhouse.

\section{LITERATURE CITED}

Akiyama, K., Matsuzaki, K.-I., and Hayashi, H. 2005. Plant sesquiterpenes induce hyphal branching in arbuscular mycorrhizal fungi. Nature 435: 824-827.

Al-Babili, S., and Bouwmeester, H. J. 2015. Strigolactones, a novel carotenoidderived plant hormone. Annu. Rev. Plant Biol. 66:161-186.

Arite, T., Umehara, M., Ishikawa, S., Hanada, A., Maekawa, M., Yamaguchi, S., and Kyozuka, J. 2009. d14, a strigolactone-insensitive mutant of rice, shows an accelerated outgrowth of tillers. Plant Cell Physiol. 50:1416-1424.

Bakker, P. A. H. M., Pieterse, C. M. J., de Jonge, R., and Berendsen, R. L. 2018. The soil-borne legacy. Cell 172:1178-1180.

Belmondo, S., Marschall, R., Tudzynski, P., López Ráez, J. A., Artuso, E., Prandi, C., and Lanfranco, L. 2017. Identification of genes involved in fungal responses to strigolactones using mutants from fungal pathogens. Curr. Genet. 63:201-213.

Berardini, T. Z., Reiser, L., Li, D., Mezheritsky, Y., Muller, R., Strait, E., and Huala, E. 2015. The Arabidopsis Information Resource: Making and mining the 'gold standard' annotated reference plant genome. Genesis 53: 474-485.

Berendsen, R. L., Vismans, G., Yu, K., Song, Y., de Jonge, R., Burgman, W. P., Burmølle, M., Herschend, J., Bakker, P. A. H. M., and Pieterse, C. M. J. 2018. Disease-induced assemblage of a plant-beneficial bacterial consortium. ISME J. 12:1496-1507.

Besserer, A., Bécard, G., Jauneau, A., Roux, C., and Séjalon-Delmas, N. 2008. GR24, a synthetic analog of strigolactones, stimulates the mitosis and growth of the arbuscular mycorrhizal fungus Gigaspora rosea by boosting its energy metabolism. Plant Physiol. 148:402-413.

Besserer, A., Puech-Pagès, V., Kiefer, P., Gomez-Roldan, V., Jauneau, A., Roy, S., Portais, J.-C., Roux, C., Bécard, G., and Séjalon-Delmas, N. 2006. Strigolactones stimulate arbuscular mycorrhizal fungi by activating mitochondria. PLoS Biol. 4:e226.

Beveridge, C. A., and Kyozuka, J. 2010. New genes in the strigolactone-related shoot branching pathway. Curr. Opin. Plant Biol. 13:34-39.

Blake, S. N., Barry, K. M., Gill, W. M., Reid, J. B., and Foo, E. 2016. The role of strigolactones and ethylene in disease caused by Pythium irregulare. Mol. Plant Pathol. 17:680-690.

Carvalhais, L. C., Rincon-Florez, V. A., Brewer, P. B., Beveridge, C. A., Dennis, P. G., and Schenk, P. M. 2019. The ability of plants to produce strigolactones affects rhizosphere community composition of fungi but not bacteria. Rhizosphere 9:18-26.

Castrillo, G., Teixeira, P. J. P. L., Paredes, S. H., Law, T. F., de Lorenzo, L., Feltcher, M. E., Finkel, O. M., Breakfield, N. W., Mieczkowski, P., Jones, C. D., Paz-Ares, J., and Dangl, J. L. 2017. Root microbiota drive direct integration of phosphate stress and immunity. Nature 543:513-518.

Cook, C. E., Whichard, L. P., Turner, B., Wall, M. E., and Egley, G. H. 1966. Germination of witchweed (Striga lutea Lour.): Isolation and properties of a potent stimulant. Science 154:1189-1190. 
Cregger, M. A., Veach, A. M., Yang, Z. K., Crouch, M. J., Vilgalys, R., Tuskan, G. A., and Schadt, C. W. 2018. The Populus holobiont: Dissecting the effects of plant niches and genotype on the microbiome. Microbiome 6:31.

De Cuyper, C., Fromentin, J., Yocgo, R. E., De Keyser, A., Guillotin, B., Kunert, K., Boyer, F.-D., and Goormachtig, S. 2015. From lateral root density to nodule number, the strigolactone analogue GR24 shapes the root architecture of Medicago truncatula. J. Exp. Bot. 66:4091.

Dor, E., Joel, D. M., Kapulnik, Y., Koltai, H., and Hershenhorn, J. 2011. The synthetic strigolactone GR24 influences the growth pattern of phytopathogenic fungi. Planta 234:419-427.

Edgar, R. C., Haas, B. J., Clemente, J. C., Quince, C., and Knight, R. 2011. UCHIME improves sensitivity and speed of chimera detection. Bioinformatics 27:2194-2200.

Flematti, G. R., Ghisalberti, E. L., Dixon, K. W., and Trengove, R. D. 2009. Identification of alkyl substituted $2 \mathrm{H}$-furo[2,3-C]pyran-2-ones as germination stimulants present in smoke. J. Agric. Food Chem. 57:9475-9480.

Foo, E., Blake, S. N., Fisher, B. J., Smith, J. A., and Reid, J. B. 2016. The role of strigolactones during plant interactions with the pathogenic fungus Fusarium oxysporum. Planta 243:1387-1396.

Foo, E., and Davies, N. W. 2011. Strigolactones promote nodulation in pea. Planta 234:1073-1081.

Foo, E., Yoneyama, K., Hugill, C. J., Quittenden, L. J., and Reid, J. B. 2013. Strigolactones and the regulation of pea symbioses in response to nitrate and phosphate deficiency. Mol. Plant 6:76-87.

Goh, C.-H., Veliz Vallejos, D. F., Nicotra, A. B., and Mathesius, U. 2013. The impact of beneficial plant-associated microbes on plant phenotypic plasticity. J. Chem. Ecol. 39:826-839.

Goodstein, D. M., Shu, S., Howson, R., Neupane, R., Hayes, R. D., Fazo, J., Mitros, T., Dirks, W., Hellsten, U., Putnam, N., and Rokhsar, D. S. 2012. Phytozome: A comparative platform for green plant genomics. Nucleic Acids Res. 40:D1178-D1186.

Graham, T. L. 1991. Flavonoid and isoflavonoid distribution in developing soybean seedling tissues and in seed and root exudates. Plant Physiol. 95:594-603.

Grant, D., Nelson, R. T., Cannon, S. B., and Shoemaker, R. C. 2010. SoyBase, the USDA-ARS soybean genetics and genomics database. Nucleic Acids Res. 38:D843-D846.

Haq, B. U., Ahmad, M. Z., Rehman, N. U., Wang, J., Li, P., Li, D., and Zhao, J. 2017. Functional characterization of soybean strigolactone biosynthesis and signaling genes in Arabidopsis MAX mutants and GmMAX3 in soybean nodulation. BMC Plant Biol. 17:259.

Kereszt, A., Li, D., Indrasumunar, A., Nguyen, C. D. T., Nontachaiyapoom, S., Kinkema, M., and Gresshoff, P. M. 2007. Agrobacterium rhizogenesmediated transformation of soybean to study root biology. Nat. Protoc. 2: 948-952.

Khan, M. M. A., and Bauer, W. D. 1988. Chemotaxis of Bradyrhizobium japonicum towards flavones and isoflavones from soybean. (Abstr. 760) Plant Physiol. 86:S-127.

Kolde, R. 2015. Pheatmap: Pretty Heatmaps. R Package Version 1.0. 8. https:// rdrr.io/cran/pheatmap/

Kozich, J. J., Westcott, S. L., Baxter, N. T., Highlander, S. K., and Schloss, P. D. 2013. Development of a dual-index sequencing strategy and curation pipeline for analyzing amplicon sequence data on the MiSeq Illumina sequencing platform. Appl. Environ. Microbiol. 79:5112-5120.

Kretzschmar, T., Kohlen, W., Sasse, J., Borghi, L., Schlegel, M., Bachelier, J. B., Reinhardt, D., Bours, R., Bouwmeester, H. J., and Martinoia, E. 2012. A Petunia $\mathrm{ABC}$ protein controls strigolactone-dependent symbiotic signalling and branching. Nature 483:341-344.

Lebeis, S. L., Paredes, S. H., Lundberg, D. S., Breakfield, N., Gehring, J., McDonald, M., Malfatti, S., del Rio, T. G., Jones, C. D., Tringe, S. G., and Dangl, J. L. 2015. Salicylic acid modulates colonization of the root microbiome by specific bacterial taxa. Science 349:860-864.

Lin, D. X., Wang, E. T., Tang, H., Han, T. X., He, Y. R., Guan, S. H., and Chen, W. X. 2008. Shinella kummerowiae sp. nov., a symbiotic bacterium isolated from root nodules of the herbal legume Kummerowia stipulacea. Int. J. Syst. Evol. Microbiol. 58:1409-1413.

Lindström, K., Murwira, M., Willems, A., and Altier, N. 2010. The biodiversity of beneficial microbe-host mutualism: The case of rhizobia. Res. Microbiol. 161:453-463.

Liu, F., Hewezi, T., Lebeis, S. L., Pantalone, V., Grewal, P. S., and Staton, M. E. 2019. Soil indigenous microbiome and plant genotypes cooperatively modify soybean rhizosphere microbiome assembly. BMC Microbiol. 19:201.

López-Ráez, J. A., Shirasu, K., and Foo, E. 2017. Strigolactones in plant interactions with beneficial and detrimental organisms: The yin and yang. Trends Plant Sci. 22:527-537.
Love, M. I., Huber, W., and Anders, S. 2014. Moderated estimation of fold change and dispersion for RNA-seq data with DESeq2. Genome Biol. 15:550.

Lundberg, D. S., Lebeis, S. L., Paredes, S. H., Yourstone, S., Gehring, J., Malfatti, S., Tremblay, J., Engelbrektson, A., Kunin, V., del Rio, T. G., Edgar, R. C., Eickhorst, T., Ley, R. E., Hugenholtz, P., Tringe, S. G., and Dangl, J. L. 2012. Defining the core Arabidopsis thaliana root microbiome. Nature 488 : 86-90.

Lundberg, D. S., Yourstone, S., Mieczkowski, P., Jones, C. D., and Dangl, J. L. 2013. Practical innovations for high-throughput amplicon sequencing. Nat. Methods 10:999-1002.

McAdam, E. L., Hugill, C., Fort, S., Samain, E., Cottaz, S., Davies, N. W., Reid, J. B., and Foo, E. 2017. Determining the site of action of strigolactones during nodulation. Plant Physiol. 175:529-542.

Mendes, L. W., Kuramae, E. E., Navarrete, A. A., van Veen, J. A., and Tsai, S. M. 2014. Taxonomical and functional microbial community selection in soybean rhizosphere. ISME J. 8:1577-1587.

Moscatiello, R., Squartini, A., Mariani, P., and Navazio, L. 2010. Flavonoidinduced calcium signalling in Rhizobium leguminosarum bv. viciae. New Phytol. 188:814-823.

Mu, Y., Jia, W.-B., Ke, Z., Zhuang, W., Wang, H.-M., Jiang, J.-D., Chen, K., and Chen, Q. 2018. Shinella pollutisoli sp. nov., isolated from tetrabromobisphenol A-contaminated soil. Int. J. Syst. Evol. Microbiol. 68: 2602-2606.

Nasir, F., Shi, S., Tian, L., Chang, C., Ma, L., Li, X., Gao, Y., and Tian, C. 2019. Strigolactones shape the rhizomicrobiome in rice (Oryza sativa). Plant Sci. 286:118-133.

Nelson, D. C., Scaffidi, A., Dun, E. A., Waters, M. T., Flematti, G. R., Dixon, K. W., Beveridge, C. A., Ghisalberti, E. L., and Smith, S. M. 2011. F-box protein MAX2 has dual roles in karrikin and strigolactone signaling in Arabidopsis thaliana. Proc. Natl. Acad. Sci. U.S.A. 108:8897-8902.

Nguyen, N. H., Song, Z., Bates, S. T., Branco, S., Tedersoo, L., Menke, J., Schilling, J. S., and Kennedy, P. G. 2016. FUNGuild: An open annotation tool for parsing fungal community datasets by ecological guild. Fungal Ecol. 20: 241-248.

Nilsson, R. H., Larsson, K.-H., Taylor, A. F. S., Bengtsson-Palme, J., Jeppesen, T. S., Schigel, D., Kennedy, P., Picard, K., Glöckner, F. O., Tedersoo, L., Saar, I., Kõljalg, U., and Abarenkov, K. 2019. The UNITE database for molecular identification of fungi: Handling dark taxa and parallel taxonomic classifications. Nucleic Acids Res. 47:D259-D264.

Olanya, O. M., and Lakshman, D. K. 2015. Potential of predatory bacteria as biocontrol agents for foodborne and plant pathogens. J. Plant Pathol. 97: 405-417.

Peláez-Vico, M. A., Bernabéu-Roda, L., Kohlen, W., Soto, M. J., and LópezRáez, J. A. 2016. Strigolactones in the Rhizobium-legume symbiosis: Stimulatory effect on bacterial surface motility and down-regulation of their levels in nodulated plants. Plant Sci. 245:119-127.

Philippot, L., Raaijmakers, J. M., Lemanceau, P., and van der Putten, W. H. 2013. Going back to the roots: The microbial ecology of the rhizosphere. Nat. Rev. Microbiol. 11:789-799.

Pieterse, C. M. J., de Jonge, R., and Berendsen, R. L. 2016. The soil-borne supremacy. Trends Plant Sci. 21:171-173.

Promputtha, I., Lumyong, S., Dhanasekaran, V., McKenzie, E. H. C., Hyde, K. D., and Jeewon, R. 2007. A phylogenetic evaluation of whether endophytes become saprotrophs at host senescence. Microb. Ecol. 53:579-590.

Rambani, A., Rice, J. H., Liu, J., Lane, T., Ranjan, P., Mazarei, M., Pantalone, V., Stewart, C. N., Jr., Staton, M., and Hewezi, T. 2015. The methylome of soybean roots during the compatible interaction with the soybean cyst nematode. Plant Physiol. 168:1364-1377.

Rehman, N. U., Ali, M., Ahmad, M. Z., Liang, G., and Zhao, J. 2018. Strigolactones promote rhizobia interaction and increase nodulation in soybean (Glycine max). Microb. Pathog. 114:420-430.

Rognes, T., Flouri, T., Ben Nichols, C. Q., and Mahé, F. 2016. VSEARCH: A versatile open source tool for metagenomics. PeerJ 4:e2584.

Roy, K. W. 1997. Fusarium solani on soybean roots: Nomenclature of the causal agent of sudden death syndrome and identity and relevance of $F$. solani form B. Plant Dis. 81:259-266.

Ruyter-Spira, C., Al-Babili, S., van der Krol, S., and Bouwmeester, H. 2013. The biology of strigolactones. Trends Plant Sci. 18:72-83.

Scherff, R. H. 1973. Control of bacterial blight of soybean by Bdellovibrio bacteriovorus. Phytopathology 63:400-402.

Schloss, P. D., Westcott, S. L., Ryabin, T., Hall, J. R., Hartmann, M., Hollister, E. B., Lesniewski, R. A., Oakley, B. B., Parks, D. H., Robinson, C. J., Sahl, J. W., Stres, B., Thallinger, G. G., Van Horn, D. J., and Weber, C. F. 2009. Introducing Mothur: Open-source, platform-independent, community- 
supported software for describing and comparing microbial communities. Appl. Environ. Microbiol. 75:7537-7541.

Segata, N., Izard, J., Waldron, L., Gevers, D., Miropolsky, L., Garrett, W. S., and Huttenhower, C. 2011. Metagenomic biomarker discovery and explanation. Genome Biol. 12:R60.

Sievers, F., and Higgins, D. G. 2018. Clustal Omega for making accurate alignments of many protein sequences. Protein Sci. 27:135-145.

Smith, S. M., and Li, J. 2014. Signalling and responses to strigolactones and karrikins. Curr. Opin. Plant Biol. 21:23-29.

Soto, M. J., Fernández-Aparicio, M., Castellanos-Morales, V., García-Garrido, J. M., Ocampo, J. A., Delgado, M. J., and Vierheilig, H. 2010. First indications for the involvement of strigolactones on nodule formation in alfalfa (Medicago sativa). Soil Biol. Biochem. 42:383-385.

Stringlis, I. A., Yu, K., Feussner, K., de Jonge, R., Van Bentum, S., Van Verk, M. C., Berendsen, R. L., Bakker, P. A. H. M., Feussner, I., and Pieterse, C. M. J. 2018. MYB72-dependent coumarin exudation shapes root microbiome assembly to promote plant health. Proc. Natl. Acad. Sci. U.S.A. 115: E5213-E5222.

Takahashi, S., Tomita, J., Nishioka, K., Hisada, T., and Nishijima, M. 2014. Development of a prokaryotic universal primer for simultaneous analysis of bacteria and archaea using next-generation sequencing. PLoS One 9:e105592.

Torres-Vera, R., García, J. M., Pozo, M. J., and López-Ráez, J. A. 2014. Do strigolactones contribute to plant defence? Mol. Plant Pathol. 15:211-216.

Untergasser, A., Cutcutache, I., Koressaar, T., Ye, J., Faircloth, B. C., Remm, M., and Rozen, S. G. 2012. Primer3-New capabilities and interfaces. Nucleic Acids Res. 40:e115.

Vaz-Moreira, I., Faria, C., Lopes, A. R., Svensson, L. A., Moore, E. R. B., Nunes, O. C., and Manaia, C. M. 2010. Shinella fusca sp. nov., isolated from domestic waste compost. Int. J. Syst. Evol. Microbiol. 60:144-148.

Wang, Q., Garrity, G. M., Tiedje, J. M., and Cole, J. R. 2007. Naive Bayesian classifier for rapid assignment of rRNA sequences into the new bacterial taxonomy. Appl. Environ. Microbiol. 73:5261-5267.

Waters, M. T., Gutjahr, C., Bennett, T., and Nelson, D. C. 2017. Strigolactone signaling and evolution. Annu. Rev. Plant Biol. 68:291-322.
Wemheuer, F., Taylor, J. A., Daniel, R., Johnston, E., Meinicke, P., Thomas, T., and Wemheuer, B. 2018. Tax4Fun2: A R-based tool for the rapid prediction of habitat-specific functional profiles and functional redundancy based on $16 \mathrm{~S}$ rRNA gene marker gene sequences. bioRxiv. doi:10.1101/490037

Wrather, J. A., Anderson, T. R., Arsyad, D. M., Tan, Y., Ploper, L. D., PortaPuglia, A., Ram, H. H., and Yorinori, J. T. 2001. Soybean disease loss estimates for the top ten soybean-producing countries in 1998. Can. J. Plant Pathol. 23:115-121.

Xie, X., Yoneyama, K., and Yoneyama, K. 2010. The strigolactone story. Annu. Rev. Phytopathol. 48:93-117.

Xu, L., Naylor, D., Dong, Z., Simmons, T., Pierroz, G., Hixson, K. K., Kim, Y.M., Zink, E. M., Engbrecht, K. M., Wang, Y., Gao, C., DeGraaf, S., Madera, M. A., Sievert, J. A., Hollingsworth, J., Birdseye, D., Scheller, H. V., Hutmacher, R., Dahlberg, J., Jansson, C., Taylor, J. W., Lemaux, P. G., and Coleman-Derr, D. 2018. Drought delays development of the sorghum root microbiome and enriches for monoderm bacteria. Proc. Natl. Acad. Sci. U.S.A. 115:E4284-E4293.

Yoneyama, K., Xie, X., Kusumoto, D., Sekimoto, H., Sugimoto, Y., Takeuchi, Y., and Yoneyama, K. 2007. Nitrogen deficiency as well as phosphorus deficiency in sorghum promotes the production and exudation of 5deoxystrigol, the host recognition signal for arbuscular mycorrhizal fungi and root parasites. Planta 227:125-132.

Yoneyama, K., Xie, X., Sekimoto, H., Takeuchi, Y., Ogasawara, S., Akiyama, K., Hayashi, H., and Yoneyama, K. 2008. Strigolactones, host recognition signals for root parasitic plants and arbuscular mycorrhizal fungi, from Fabaceae plants. New Phytol. 179:484-494.

Zhalnina, K., Louie, K. B., Hao, Z., Mansoori, N., Nunes da Rocha, U., Shi, S., Cho, H., Karaoz, U., Loqué, D., Bowen, B. P., Firestone, M. K., Northen, T. R., and Brodie, E. L. 2018. Dynamic root exudate chemistry and microbial substrate preferences drive patterns in rhizosphere microbial community assembly. Nat. Microbiol. 3:470-480.

Zhang, B., Zhang, J., Liu, Y., Shi, P., and Wei, G. 2018. Co-occurrence patterns of soybean rhizosphere microbiome at a continental scale. Soil Biol. Biochem. 118:178-186. 